\title{
Abstract
}

Objectives: 1) To identify risk factors for chronic disability in people with acute whiplash associated disorders (WAD). 2) To estimate the impact of the numbers of risk factors present. Design: Prospective cohort study. Data were collected, on average, 32 days after injury $(S D=10.9)$ and 12 months later. Baseline measures of pain, disability, range of movement and psychological and behavioural factors were independent variables and chronic disability at 12 months was the dependent variable in a multivariable logistic regression analysis.

Setting: National Health Service physiotherapy departments.

Participants: Participants ( $n=599$ ) with symptoms at least 3 weeks after injury, self-referred to receive physiotherapy as part of a pragmatic randomised controlled trial. 430 (72\%) participants provided complete data for this analysis.

Main outcome measures: Chronic disability based on Neck Disability Index scores.

Results: 136 (30\%) participants developed chronic disability. High baseline disability (OR 3.3, $95 \% \mathrm{Cl} 1.97-5.55)$, longer predicted recovery time (OR $2.4,95 \% \mathrm{Cl} 1.45-3.87$ ), psychological distress (OR 1.9, 95\% Cl 1.05-3.51), greater use of passive coping (OR 1.8, 95\% Cl 1.07-2.97) and greater number of symptoms $(\mathrm{OR} 1.7,95 \% \mathrm{Cl} 1.07-2.78)$ were associated with chronic disability. One risk factor resulted in 3.5 times the risk $(95 \% \mathrm{Cl} 1.04-11.45)$ of chronic disability but this risk increased to 16 times $(95 \% \mathrm{Cl} 5.36-49.27)$ in those with four or five risk factors.

Conclusion: Baseline disability had the strongest association with chronic disability but psychological and behavioural factors were also important. Treatment strategies should reflect this which may require a change in the current physiotherapy approach to acute WAD. The total number of risk factors present should be considered when evaluating the potential for poor outcome.

Key words: neck injuries; whiplash injuries, rehabilitation; physiotherapy; disability, pain

\section{Risk factors for chronic disability in a cohort of patients with acute whiplash associated disorders seeking physiotherapy treatment for persisting symptoms.}

\section{Introduction}

Whiplash Associated Disorders (WAD) describe the symptoms experienced following a whiplash injury (1). WAD are a significant public health problem as it is estimated that only 
$50 \%$ of individuals who develop WAD recover fully and there remains uncertainty as to the reasons for this (2).

This prospective cohort study investigated if psychological and physical factors were risk factors for the development of chronic disability following an acute whiplash injury. We were interested in identifying risk factors that were potentially modifiable and could be treatment targets for physiotherapy management. We undertook a series of systematic reviews to synthesise evidence of existing risk factors, to identify areas of uncertainty, and methods of measurement $(3,4)$. In terms of psychological and behavioural factors, we concluded from the reviews that there was evidence that low self-efficacy and elevated scores on the Impact of Events Scale (post traumatic distress) were associated with chronic disability but evidence supporting other factors was less clear (fear avoidance, catastrophising, coping, depression and anxiety) (4). Expectations of outcome and treatment preferences had yet to be investigated in acute WAD when the systematic reviews were conducted but warranted investigation. We found evidence that age, gender, initial injury severity and a history of previous neck pain were associated with poor outcome in acute $\operatorname{WAD}(2,3,5)$ but evidence for physical measures such as range of movement was inconclusive (3) and needed further investigation.

Most prospective cohort studies view risk factors in isolation, however, Carroll (6) emphasises that risk factors do not work alone. Little work has been published on understanding the impact of multiple risk factors. There is a need to further our understanding to aid the development of more effective treatments for patients with acute WAD.

Therefore, the two aims of this paper were:

1. To identify risk factors for developing chronic disability in a cohort of patients with acute WAD seeking physiotherapy treatment for persistent symptoms at least 3 weeks post injury.

2. To estimate the impact of the number of risk factors present at baseline on outcome. 


\section{Methods}

\subsection{Patient Sample}

All participants had sustained an acute whiplash injury and were experiencing neck pain at least 3 weeks after injury. Participants were part of a large cohort of patients who attended NHS Emergency Departments (ED) following their whiplash injury and had then self-referred to receive physiotherapy as part of a randomised controlled trial (7). Participants were randomly allocated to one of two physiotherapy treatments (a package of physiotherapy treatment or an advice session) (8). Individuals were eligible if they had sustained a whiplash injury of WAD grade I-III [Quebec Taskforce grading system (1)] within the last 6 weeks, reported neck symptoms in the previous 24 hours, had not sustained any fractures or loss of consciousness at the time of the injury/ED presentation, were not hospitalised, had no contraindications to physiotherapy treatment and were 18 years or older.

\subsection{Data collection}

Participants attended a research clinic where they provided written consent to take part in the study, completed a questionnaire and a standardised examination was undertaken by research staff. Follow up was carried out 12 months after ED attendance by masked postal questionnaire. Participants were recruited from January 2006 until November 2007.

\subsection{Baseline measurements}

A large data set was collected at baseline (Table 1). Variable selection was informed by our systematic literature reviews $(3,4)$, the broader pain literature and underpinned by the biopsychosocial model of pain (9). Variables were grouped in 3 categories: injury severity and range of movement (these are elements commonly assessed by physiotherapists in clinical practice), psychological and behavioural factors, pre-existing factors. Treatment allocation was also included in the analysis as participants were taking part in a randomised controlled trial (RCT). 
We searched the literature to identify appropriate methods of data collection. Validated questionnaires with acceptable levels of reliability were chosen where possible. Some questions were designed for the study when there was no suitable questionnaire available (e.g. predicted time to recovery). Overburdening participants was a concern, so short versions of questionnaires were used if available (e.g. Coping Strategy Questionnaire) or a single question was designed to measure the construct of interest (e.g. ability to cope).

\subsubsection{Injury severity and range of movement}

Baseline disability was measured by the Neck Disability Index (NDI) (10) . Participants rated their pain intensity using the Modified Von-Korff Pain Scale (11) which produces a single score encompassing current pain, average pain and worst pain in the last week. The Quebec Taskforce WAD grading system (1) was used to grade injury severity in the ED by the treating clinician and at the time of enrolment in the study. Participants also indicated the presence of 15 symptoms related to their whiplash injury using the physical symptoms subscale from the Cervical Spine Outcome Questionnaire (12).

Neck range of movement (ROM) were taken using the cervical ROM device (13). The participant was assessed in a seated position and measures of cervical flexion, extension, lateral flexion and rotation were taken in a standardised manner according to a protocol. A total ROM was calculated by adding the measurements together.

\subsubsection{Psychological and behavioural factors}

The use of active and passive coping was measured using the short version ( 7 items) of the Coping Strategies Questionnaire (CSQ) (14). The items were grouped into two subscales representing the use of passive coping strategies ( 2 items) and active coping strategies ( 5 items) (15). The Pain Catastrophising Scale (PCS) was used to measure the frequency with 
which an individual expresses catastrophic thoughts when they are in pain (16). The physical activity subscale of the Fear Avoidance Beliefs Questionnaire (FABQ) was used to assess fear avoidance (beliefs about the effect of physical activity on their pain) (17). The words "back pain" were replaced with "neck pain". Psychological distress was measured using the General Health Questionnaire-12 (18) which consists of 12 items that assess the severity of different problems over the past few weeks. The standard scoring system was used in this study resulting in a score out of 12 (18). The Impact of Events Scale (IES) was used to measure post-traumatic distress (19) where participants report the frequency of 15 symptoms in the past 7 days.

A single question prior to randomisation was used to elicit participants' preferences for the treatments being evaluated in the RCT according to recommendations by the Preference Collaborative Review Group (20). Participants were asked to provide a self-assessment of how long they thought they would take to recover from their injury. This predicted time to recovery was measured by asking participants to select from 6 different time frames. The response categories chosen represent clear and distinct timeframes that participants could distinguish from each other (Table 1). Likert scales were used to measure participants endorsement that neck exercises are helpful following neck injury, their ability to cope with their neck problem (self-efficacy) and the expected benefit of the treatment they were to receive (21).

\subsubsection{Pre-existing factors}

Pre-existing factors with potential to influence outcome were collected including age, gender, social support (22), the presence of pre-existing neck problems and chronic widespread pain (23).

\subsection{Outcome}

Chronic disability was defined as a score of $\geq 30 \%$ on the NDI at 12 months follow up. A score of $\geq 30 \%$ represents moderate, severe or complete disability according to the NDI categories published by Vernon and Mior which have been used in previous research $(10,24)$. A binary 
outcome was used to facilitate use within clinical practice by providing a defined cut off point for clinicians to identify patients with on-going difficulties.

\subsection{Sample size}

It is recommended that a minimum of 10 participants classified as the outcome of interest (developed chronic disability) are needed for each variable included in a logistic regression model (25). Based on this assumption the final logistic regression model was large enough to include 13 variables as 136 participants were classified as being chronically disabled at follow up.

\subsection{Statistical methods}

The primary analysis was logistic regression. Baseline factors were dichotomised using established cut-offs where available (Table 1 ) to aid interpretation and application to clinical practice (26). Where no published cut-offs existed tertile splits were used to dichotomise the data. Participants who scored in the lower or upper third of responses for a particular measure were compared to the rest of participants. This was dependent on the variable of interest. For example, age was dichotomised using the upper third as the cut-point but ROM used the lower third.

To avoid over-fitting the final model we carried out a series of analyses. We assessed the univariate relationship (odds ratios) between each baseline variable and outcome to identify factors for the multivariable analyses. A conservative level of significance was set at $p<0.25$ to select these factors (27). We then constructed a logistic regression model for each grouping of baseline variables outlined in Section 2.3 and from these we selected the factors to go into the final model. In each analysis, the baseline variables outlined in Section 2.3 were the independent variables and the presence of chronic disability at follow up was the dependent variable. Multivariable analysis was carried out using a forward, stepwise logistic regression model (28) with a significance level set at $p<0.05$ to retain factors. Factors found to have a significant relationship with chronic disability in each of these models were then entered into the final logistic regression model which also included treatment allocation. 
Participants were included in the multivariable analysis if they had complete data for each variable included in the analysis.

The final model was checked for multicollinearity. Multicollinearity occurs when two or more predictor variables are highly correlated (26). This is problematic because if two predictors are closely related to each other then it is impossible to accurately estimate the unique contribution that each factor makes to the regression model and may be a source of bias in the model (26). We calculated variance inflation factors (VIF), tolerance and collinearity diagnostics (eigenvalues, condition index and variance proportions) to check they fell within the recommended limits, which would indicate that multicollinearity was not present in the model (26).

Following the final logistic regression analysis, the number of risk factors present at baseline for each participant was calculated. A Chi squared test for trend was performed to assess if there was a dose like relationship between the number of risk factors present and outcome (27). The relative risk of developing chronic disability according to the number of risk factors present was then calculated (no risk factors as the comparator).

Baseline data from responders and non-responders were compared to identify any difference between the two groups using an independent t-test. Statistical Package for the Social Sciences (IBM, New York) V15.0 was used to carry out all statistical analyses.

\section{Results}

599 participants provided baseline data (Table 1) which was collected, on average, 32 days after injury $(S D=10.9$, range $=7-74) .459$ participants $(76.6 \%)$ returned a complete NDI questionnaire at 12 months. 29 participants (4.8\%) were excluded from the analysis due to missing baseline data resulting in $430(71.8 \%)$ participants contributing data to the final logistic regression model. The average age of those responding to 12 month follow up was 41 years , the majority were female (298/459 [65\%]) with a WAD grade II whiplash injury 
(337/459 [73\%]) and an average NDI score of $41 \%$ (SD=16.1). Most notably, non-responders were younger, reported higher mean baseline disability at baseline and reported more catastrophic thinking than responders but the differences were small (Online Table).

\subsection{Predictors of chronic disability}

Approximately $30 \%$ of participants ( $n=136 / 459)$ who returned their 12 month questionnaire had developed chronic disability. Baseline disability, pain intensity and use of passive coping strategies had the strongest univariate associations with chronic disability. The separate models constructed for injury severity and ROM (Model 1), psychological and behavioural factors (Model 2) and pre-existing factors (Model 3) are reported in Table 2, along with the findings from the final logistic regression model. Only those factors with a significant association with chronic disability are shown.

Five baseline factors were associated with increased risk of chronic disability. A high level of baseline disability ( $\geq 50 \%$ on the NDI) was associated with 3.3 times the odds of developing chronic disability compared to those participants with lower baseline scores. This risk factor was present in $30 \%(n=181)$ of participants. A predicted recovery time of 6 months or more was also associated with considerably increased risk at 2.4 times the odds of developing chronic disability compared to those who were more optimistic. 31\% ( $n=182)$ of participants presented with this risk factor. More modest increases in risk were seen with the remaining risk factors. Participants reporting psychological distress ( $\geq 4$ on the GHQ-12) [n=417(70\%)] were just less than twice the odds of developing chronic disability compared to those who were not psychologically distressed. Participants who used passive coping strategies more frequently (scoring $\geq 5$ on the passive coping subscale of the PCQ) compared to those with less frequent use were 1.77 the odds of developing chronic disability. This risk factor was present in $37 \%$ ( $n=220)$ of participants. Participants reporting 6 or more physical symptoms $[n=220(38 \%)]$ were also at greater risk of chronic disability (odds 1.72 times greater) compared to those with fewer symptoms. 
VIF, tolerance and collinearity diagnostics (eigenvalues, condition index and variance proportions) were examined and all were within the recommended limits indicating that there was no multicollinearity present in the final model.

\subsection{Relative risk of chronic disability based on the number of risk}

\section{factors present}

The presence of high baseline disability was the strongest predictor of disability but rarely occurred in isolation as it was the sole risk factor in only 2 participants $(<1 \%)$.

Approximately $60 \%$ of the cohort presented with more than one risk factor (Figure 1). Only $23(4 \%)$ participants presented with all 5 risk factors so participants with 4 or 5 risk factors were presented as a single category. Participants with no risk factors were the comparator group and had a $4 \%$ ( 1 in 25 ) chance of developing chronic disability. With the addition of each risk factor there was an increase in the relative risk of developing chronic disability compared to those with no risk factors (Figure 1). The increase in risk ranged from 3.5 times the risk for those with one risk factor up to 16 times the risk for those with 4-5 risk factors. The Chi squared test for trend confirmed there was a dose like response relationship and as the number of risk factors increased the proportion of participants with chronic disability also increased $(p<0.001)$.

\section{Discussion}

Baseline measures of disability and symptoms along with psychological and behavioural factors were identified as risk factors for chronic disability. As the number of risk factors present at baseline increased so did the risk of chronic disability.

The factors selected for testing were underpinned by a biopsychosocial model of disability. We grouped together a number of factors to represent the "bio" aspect of this model (labelled injury severity and ROM) that would be traditionally assessed by physiotherapists in clinical practice. Our findings agreed with previous research that patients reporting high level 
of disability/pain early on after injury are at risk of poor outcome following a whiplash injury. This study supports the consistent assertion that there is association between greater injury severity and chronic disability $(2,5)$.

We also examined a group of factors labelled "psychological and behavioural factors" that represented the "psycho" aspect of the biopsychosocial model and may not be commonly assessed in patients with acute injuries by physiotherapists. The psychological factor associated with the greatest risk of chronic disability was self-assessed predicted time to recovery which measures expectations of recovery. Expectations are thought to result in neurophysiological changes within the nervous system as well as behavioural changes that impact on how an individual will manage their condition $(29,30)$. There is evidence that expectations of recovery can influence outcome in WAD (31) but this is the first study to demonstrate that individuals who believe they will take a long time to recover are at risk of chronic disability.

Psychological distress was common. We measured this using the GH-12 which primarily assesses anxiety and depression (18). Previous findings are mixed regarding the role of anxiety and depression in the development of chronic disability in acute WAD (4). The GHQ-12 measures psychological state at the point of assessment compared to the "usual" state so it does not measure chronic psychological problems. This was important in the context of this study as we aimed to assess participants' psychological status compared to their usual state (i.e. pre-injury). We used published cut points for a UK population to identify psychological morbidity (18). This study provides evidence that psychological distress present early after injury is a risk factor for chronic disability and therefore clinicians should be aware of the impact of anxiety and depression on outcome.

Use of passive coping strategies was a behavioural factor that influenced outcome. In this study, coping was conceptualised using an active-passive approach. Active strategies involve an attempt by the patient to deal with the pain themselves (15). Passive strategies are characterised by helplessness and/or reliance on others (15). Active coping strategies are 
considered helpful to recovery while passive strategies are viewed as ineffective. However, only passive coping was found to be related to chronic disability. Greater use of passive coping has also been associated with longer recovery time in acute WAD (32) suggesting that the use of passive coping is a more important concept to consider when evaluating potential for poor outcome.

The influence of psychological and behavioural factors could be considered unimportant compared to baseline disability which had the strongest association with chronic disability. However, estimating an individual's risk of chronic disability based solely on baseline disability may result in underestimation of the risk, as it rarely occurs in isolation. For participants without baseline risk factors, only $4 \%$ developed chronic disability compared to those participants with four and five risk factors where $67 \%$ developed chronic disability. This suggests that even when the increased risk associated with one risk factor is relatively small when combined with other factors it can have a greater impact. The confidence intervals for the risk ratios were wide suggesting some uncertainty about the precision of these estimates. Even if the lower limit of the $95 \%$ confidence interval is considered for those with 4 or 5 risk factors, a substantial increase in the risk of chronic disability is demonstrated. The additive effect of increasing numbers of risk factors has been demonstrated in one previous study (33).

\section{Clinical implications}

The evidence to date is that traditional physiotherapy approaches provide limited benefit to patients following a whiplash injury $(7,34)$. There have been calls for a greater focus on the management of psychosocial aspects of acute WAD (35). Treatments targeting disability and symptoms early on through pharmacological, psychological and behavioural approaches need to be developed. High levels of pain and disability are consistently identified as risk factors for chronic disability following a whiplash injury so effective pain relief should be considered a priority for treatment. Psychological and behavioural factors were identified as risk factors for poor outcome over factors such as neck ROM that are routinely assessed and targeted in clinical practice. This suggests a need for a shift in traditional physiotherapeutic 
approaches away from physical interventions commonly used to manage acute conditions to psychological and behavioural approaches as we have observed in chronic pain conditions. Psychological distress was an independent risk factor for chronic disability but it is likely to be partly related to the symptoms and disability reported by the patient. Physiotherapists have an important role to play in providing reassurance to patients to allay anxiety and equipping patients with strategies for reducing symptoms and avoiding the use of ineffective passive coping strategies.

Importantly, this study also showed that participants with no risk factors were at a very low risk of chronic disability. Physiotherapists can reassure these patients they that should expect a good recovery with minimal intervention.

\section{Methodological issues}

We chose to dichotomise baseline factors to make it easier for clinicians to apply these findings to clinical practice. Dichotomising variables does reduce the power for detecting relationships between variables (26) and is one reason that only $19 \%-28 \%$ of the variance was explained in the final model. The models presented may have been improved by the inclusion of additional measures. For example, the presence of cold hyperalgesia has been identified as a risk factor (24) but measuring this was beyond the scope of this research due to the already considerable burden of data collection on participants.

This was an exploratory analysis to identify targets for physiotherapy treatment. We acknowledge that further work is needed to develop screening tools that accurately identify those at risk of chronic disability although Sterling and colleagues are making progress in this field (36). The factors identified in this study are not definitive but add to the growing body of evidence regarding the development of chronic disability in patients with acute WAD.

A strength of this study was the large sample size so, even with missing data, the sample size is sufficient. The differences between responders and non-responders were small and 
unlikely to have influenced outcome. For example, the difference in NDI scores, although statistically significant, was less than the clinically important difference estimated to be 5 points (37).

\section{External validity}

The trial from which these data were derived was extremely large, from several geographic areas, was very pragmatic in its cluster design and included a spread of ages and injury severity which increase the likelihood of it being generalizable to a wider population. Participants had persistent symptoms for which they sought physiotherapy and presented with symptoms commonly reported by patients following a whiplash injury (38). Participants are likely to be similar to patients who seek physiotherapy in both primary and secondary care health settings making these findings highly applicable to clinicians and their patients. The issue of generalization is likely to be similar for any observational design that requires patient consent for participation.

\section{Further research}

The best way to utilise knowledge about risk factors is still unclear (39). It is unknown if targeting these factors will improve outcomes. The number of risk factors could guide treatment planning with more intensive treatments, such as cognitive behavioural interventions, provided for those with more risk factors. This type of stratified approach for patients with LBP has proved to be effective $(40)$ but needs to be evaluated in WAD. Initial work has begun on this approach but it remains an area requiring further development (41).

In conclusion, baseline disability had the strongest association with chronic disability but psychological and behavioural factors were also important. Treatment strategies should reflect this which may require a change in the current physiotherapy approach to acute WAD. The total number of risk factors present should be considered when evaluating the potential for poor outcome. 


\section{Ethical Approval}

Ethical approval was given by the Trent Multicentre Research Ethics Committee (reference MREC/04/4/003).

\section{Acknowledgements}

We would like to acknowledge the research clinicians who collected baseline data for this study: Liz Ball, Janet Lowe and Cheryl Ritchie (University of Warwick); Claire Hunt, Rachel Trickey and Elaine Willmore (Cheltenham General and Royal Gloucester Hospitals); Heather Shilliday (Countess of Chester Hospital); Susan Kempson (Frenchay Hospital); Bridget Gray and Elizabeth Oastler (John Radcliffe Hospital); Helen Harrison (Kettering General Hospital); Melissa Minshull and Kate Rees (Royal Gwent Hospital, Newport); Steve Lodge (Stoke Mandeville and High Wycombe Hospital). We would also like to thank Emma Withers (Trial Co-ordinator) with her assistance in accessing data that was used in the study and Dr Chris Bridle who provided PhD supervision for EW.

\section{Conflicts of interest statement}

No conflict of interest to declare.

\section{Funding sources}

Financial support for this work was provided as part of funding for the Managing Injuries of the Neck Trial by the NIHR Health Technology Assessment Programme (HTA number 02/35/02). Trial registration: ISRCTN33302125, http://www.isrctn.org/. The sponsor had no role in the design, conduct, analysis and interpretation of data, writing the manuscript or the decision to submit the manuscript for publication. 
This project benefited from facilities funded through Birmingham Science City Translational Medicine Clinical Research and Infrastructure Trials Platform, with support from Advantage West Midlands (AWM).

\section{References}

1. Spitzer WO, Skovron ML, Salmi LR, Cassidy JD, Duranceau J, Suissa S, et al. Scientific monograph of the Quebec Task Force on Whiplash-Associated Disorders: redefining "whiplash" and its management. Spine. 1995;20(8) Suppl(8):1S-73S.

2. Carroll L, Holm L, Hogg-Johnson S, Côté P, Cassidy J, Haldeman S, et al. Course and prognostic factors for neck pain in whiplash-associated disorders (WAD): results of the Bone and Joint Decade 2000-2010 Task Force on Neck Pain and Its Associated Disorders. Spine. 2008;33(4 Suppl):S83-92.

3. Williams M, Williamson E, Gates S, Lamb S, Cooke M. A systematic literature review of physical prognostic factors for the development of Late Whiplash Syndrome. Spine. 2007;32(25):E764-80.

4. Williamson E, Williams M, Gates S, Lamb S. A systematic literature review of psychological factors and the development of late whiplash syndrome. Pain. 2008;135(1-2):20-30.

5. Walton DM, Macdermid JC, Giorgianni AA, Mascarenhas JC, West SC, Zammit CA. Risk factors for persistent problems following acute whiplash injury: update of a systematic review and meta-analysis. J Orthop Sports Phys Ther. 2013;43(2):31-43.

6. Carroll L. Beliefs and expectations for recovery, coping, and depression in whiplashassociated disorders: lessening the transition to chronicity. Spine (Phila Pa 1976). 2011;36(25 Suppl):S250-6.

7. Lamb SE, Gates S, Williams MA, Williamson EM, Mt-Isa S, Withers EJ, et al. Emergency department treatments and physiotherapy for acute whiplash: a pragmatic, twostep, randomised controlled trial. Lancet. 2013;381(9866):546-56.

8. Williamson E, Williams M, Hansen Z, Joseph S, Lamb S. Development and delivery of a physiotherapy intervention for the early management of whiplash injuries: the Managing Injuries of Neck Trial (MINT) Intervention. Physiotherapy. 2009;95(1):15-23.

9. Pincus T, Kent P, Bronfort G, Loisel P, Pransky G, Hartvigsen J. Twenty-five years with the biopsychosocial model of low back pain-is it time to celebrate? A report from the twelfth international forum for primary care research on low back pain. Spine (Phila Pa 1976). 2013;38(24):2118-23.

10. Vernon H. The Neck Disability Index: state-of-the-art, 1991-2008. J Manipulative Physiol Ther. 2008;31(7):491-502.

11. Underwood MR, Barnett AG, Vickers MR. Evaluation of two time-specific back pain outcome measures. Spine. 1999;24(11):1104-12.

12. BenDebba MHJ, Ducker TBEJM. Cervical spine outcomes questionnaire. SPINE. 2002;27(19):2116-24.

13. Williams MA, Williamson E, Gates S, Cooke MW. Reproducibility of the cervical range of motion (CROM) device for individuals with sub-acute whiplash associated disorders. Eur Spine J. 2012;21(5):872-8.

14. Jensen MP, Keefe FJ, Lefebvre JC, Romano JM, Turner JA. One- and two-item measures of pain beliefs and coping strategies. Pain. 2003;104(3):453-69.

15. Snow-Turek AL, Norris MP, Tan G. Active and passive coping strategies in chronic pain patients. Pain. 1996;64(3):455-62.

16. Sullivan MJL, Bishop SR, Pivik J. The Pain Catastrophizing Scale: Development and validation. Psychological Assessment. 1995;7(4):524-32. 
17. Cleland J, Fritz J, Childs J. Psychometric properties of the Fear-Avoidance Beliefs Questionnaire and Tampa Scale of Kinesiophobia in patients with neck pain. Am J Phys Med Rehabil. 2008;87(2):109-17.

18. Goldberg D, Williams P. A User's Guide to the General health Questionnaire. Windsor: NFER; 1988.

19. Joseph S. Psychometric evaluation of Horowitz's Impact of Event Scale: a review. Journal of traumatic stress. 2000;13(1):101-13.

20. Group PCR. Patients' preferences within randomised trials: systematic review and patient level meta-analysis. BMJ. 2008;337.

21. Devilly G, Borkovec T. Psychometric properties of the credibility/expectancy questionnaire. Journal of Behaviour Therapy and Experimental Psychology. 2000;31:73-86.

22. Zimet GD, Dahlem NW, Zimet SG, Farley GK. The Multidimensional Scale of Perceived Social Support. Journal of personality assessment. 1988;52(1):30-41. awrene Erbaum. 23. Hunt I, Silman A, Benjamin S, McBeth J, Macfarlane G. The prevalence and associated features of chronic widespread pain in the community using the 'Manchester' definition of chronic widespread pain. Rheumatology (Oxford). 1999;38(3):275-9.

24. Sterling M, Jull G, Vicenzino B, Kenardy J, Darnell R. Physical and psychological factors predict outcome following whiplash injury. Pain. 2005;114(1-2):141-8.

25. Peduzzi P, Concato J, Kemper I, Holford T, Feinstein A. A Simulation study of the number of events per variable in logistic regression analysis. J Clin Epidemiol. 1996;49(12):1373-9.

26. Szklo M, Nieto FJ. Epidemilogy: Beyond the Basics. Anonymous, editor. Gaithersburg, Maryland, USA: Apsen Publication; 2000.

27. Altman D. Practical statistics for medical research. London: Chapman \& Hall/CRC; 1991.

28. Field A. Discovering statistics using SPSS. 3rd edition ed. London: SAGE Publications; 2009.

29. Wiech K, Ploner M, Tracey I. Neurocognitive aspects of pain perception. Trends Cogn Sci. 2008;12(8):306-13.

30. Janzen J, Silvius J, Jacobs S, Slaughter S, Dalziel W, Drummond N. What is a health expectation? Developing a pragmatic conceptual model from psychological theory. Health Expect. 2006;9(1):37-48.

31. Carroll L, Holm LW, Ferrari R, Ozegovic D, Cassidy JD. Recovery in whiplashassociated disorders: do you get what you expect? J Rheumatol. 2009;36(5):1063-70.

32. Carroll $\amalg$, Cassidy JD, Cote P. The role of pain coping strategies in prognosis after whiplash injury: Passive coping predicts slowed recovery. Pain. 2006;124(1-2):18-26.

33. Atherton K, Wiles NJ, Lecky FE, Hawes SJ, Silman AJ, Macfarlane GJ, et al. Predictors of persistent neck pain after whiplash injury. Emergency medicine journal : EMJ. 2006;23(3):195-201.

34. Hurwitz E, Carragee E, van der Velde G, Carroll L, Nordin M, Guzman J, et al. Treatment of neck pain: noninvasive interventions: results of the Bone and Joint Decade 2000-2010 Task Force on Neck Pain and Its Associated Disorders. Spine. 2008;33(4 Suppl):S123-52.

35. Côté $P$, Soklaridis S. Does early management of whiplash-associated disorders assist or impede recovery? Spine (Phila Pa 1976). 2011;36(25 Suppl):S275-9.

36. Sterling M, Hendrikz J, Kenardy J, Kristjansson E, Dumas JP, Niere K, et al. Assessment and validation of prognostic models for poor functional recovery 12 months after whiplash injury: a multicentre inception cohort study. Pain. 2012;153(8):1727-34.

37. MacDermid J, Walton D, Avery S, Blanchard A, Etruw E, McAlpine C, et al. Measurement properties of the neck disability index: a systematic review. J Orthop Sports Phys Ther. 2009;39(5):400-17.

38. Jull G, Sterling M, Falla D, Treleaven J, O'Leary S. Whiplash, Headach, and Neck Pain. Edinburgh: Churchill Livingstone; 2008. 
39. Sterling M, Carroll LJ, Kasch H, Kamper SJ, Stemper B. Prognosis after whiplash injury: where to from here? Discussion paper 4. Spine (Phila Pa 1976). 2011;36(25 Suppl):S330-4.

40. Hill JC, Whitehurst DG, Lewis M, Bryan S, Dunn KM, Foster NE, et al. Comparison of stratified primary care management for low back pain with current best practice (STarT Back): a randomised controlled trial. Lancet. 2011;378(9802):1560-71.

41. Jull G, Kenardy J, Hendrikz J, Cohen M, Sterling M. Management of acute whiplash: A randomized controlled trial of multidisciplinary stratified treatments. Pain.

2013;154(9):1798-806. 